\title{
A study of application of QT interval correction and QT/RR regression models to ECG recording in Diabetes Mellitus
}

\author{
Purushothaman. G, Chandrasekhar. M, Qairunnisa. S, Madhuri. B. A., \\ Suresh. M, Ambareesha Kondam \\ Department of Physiology, Meenakshi Medical College \& Research Institute. \\ Enathur, Kanchipuram - 631 552. Tamil Nadu, India
}

\begin{abstract}
Introduction Patients with Diabetes Mellitus (DM) are at increased risk of developing cardiovascular complications and autonomic dysfunction. The current study focuses on the $Q T$ interval and QT/RR regression models on Diabetic patients, which will help in identifying the patients who are likely to develop cardiac arrhythmias and autonomic neuropathy.

Material and Methods This study was conducted in the Department of Physiology, MMCH\&RI. Sixty subjects were included in this study after obtaining permission from the Institutional Human Ethical Committee. The study group consisted of $66 \%$ of males and $34 \%$ of females who were in the age group of 30 to 65 years. ECG was recorded in DM as well as in normal subjects. The QT and RR intervals were measured in Lead II. The observed QT interval was corrected for heart rate according to Bazett's formula. The Subjects were studied using linear QT/RR regression models to assess cardiovascular abnormalities. The autonomic function tests (AFT) were studied on the patients which included heart rate variability during deep breathing, Valsalva ratio \& blood pressure response to hand grip etc.,

Results The results were analyzed using SPSS statistical software with non-parametric Chi square test and the significance level was fixed at $P<0.05$. There was a significant difference in QTc of DM $(0.446 \pm$

0.03 in $\mathrm{ms})$ when compared with normal subjects $(0.412 \pm 0.012$ in $\mathrm{ms})$. When the duration of diabetes was taken into account, the QTc (ms) deviations as obtained by the QT/RR linear regression models differed significantly $\left(R^{2}=0.009\right)$ for diabetes when compared with normal subjects. Abnormal responses on individual AFT revealed that HRV during deep breathing \& Valsalva ratio $(0.99 \pm 0.08$ and $1.07 \pm 0.09$ respectively) were more significant whereas 30: 15 ratio and handgrip test were less significant. SBP response to standing was shown to be significant $(147 \pm 10 \mathrm{in} \mathrm{mmHg})$ whereas $\mathrm{DBP}(86 \pm 10 \mathrm{in} \mathrm{mmHg}) \mathrm{did}$ not show any difference.

Conclusion In conclusion, the analysis of QT intervals and its deviations attained by the linear regression model, differed significantly for Diabetes with longer duration. If it is further validated with the other regression model, it could be used to predict the increasing risk factor of Cardio autonomic neuropathy.
\end{abstract}

Keywords-QT interval, $Q T / R R$ ratio, $Q T$ interval correction and variability, Diabetes Mellitus, Cardiovascular complications.

\section{INTRODUCTION}

Diabetes mellitus (DM) comprises a group of common metabolic disorders that causes the increasing risk and mortality among diabetic patients and typically occurs in the cardiovascular system. Number of non invasive test has been used in the diagnosis of DM to find the incidence of Cardiac Autonomic Neuropathy. QT interval reflects the total duration of ventricle myocardial depolarization and repolarization. QT prolongation has been shown to predict Cardiac death in DM. Assessment of QT interval could be cost effective way identifying DM with risk of cardiovascular complication and sudden death. This current study focused on the QT interval and QT/RR regression models on Diabetic patients, which will help in identifying the patients who are at the risk to development Cardiac arrhythmias and Autonomic neuropathy.

\section{MATERIAL AND METHODS}

Sixty subjects were included in this study after obtaining permission from the Institutional Human Ethical Committee. ECG was recorded in 30 normal and 30 middle aged diabetic patients and was divided under two groups. The Study period was spread over the period of two years 2009-2011. 67 subjects were assessed and 30 were selected based on the following protocol. 


\section{PROTOCOL OF THE STUDY}

To establish the normal ranges for assessing autonomic dysfunction a battery of 5 cardiovascular reflex tests were performed by means of computerized portable system in the above mentioned groups as recommended by Ewing et. al. These include HRV during deep breathing, 30:15 ratio on standing, Valsalva ratio, BP response to Standing and Hand grip test. In these tests out of which atleast two must be abnormal for defined diagnosis of CAN and QT interval was calculated by RMS Polyrite. The QTc was determined with Bazett's formula $(\mathrm{QTc}=\mathrm{QR} / \sqrt{\mathrm{RR}})$ and value more than $0.44 \mathrm{sec}$ was considered as prolonged. In order to study the QT/RR relation, the data of QT and RR interval of each subject were studied and the linear regression model $(\mathrm{QT}=\beta+\alpha \times \mathrm{RR})$ was used to test the patient data.

\section{STATISTICAL ANALYSIS}

The data was analyzed using the SPSS software programme. Non-parametric test-Mann-Whitney U test was used to compare the two groups with respect to the different risk factors considered in the study example. A p value $<0.05$ was considered statistically significant.

\section{RESULTS}

This study include 60 subjects, 30 in the study group and 30 in control group were examined and recruited into the study.

Table 1: Male and Female ratio

\begin{tabular}{|l|l|l|}
\hline Gender & Study $(\mathbf{n}=\mathbf{3 0})$ & Control $(\mathbf{n}=\mathbf{3 0})$ \\
\hline Male & $19(63.3 \%)$ & $16(53.3 \%)$ \\
\hline Female & $11(36.7 \%)$ & $14(46.7 \%)$ \\
\hline
\end{tabular}

30 subjects of the study constitute 16 males and 14 females (male: female ratio 1.42:1) and were in the age range from 25 to 65 years.

\begin{tabular}{|c|c|c|c|c|}
\hline Risk Factors & \multicolumn{3}{|l|}{ Groups } & $\begin{array}{l}\text { Significant } \\
\text { P value }\end{array}$ \\
\hline $\begin{array}{l}\text { Duration } \\
\text { Diabetes }\end{array}$ & $\begin{array}{l}<2 \mathrm{yrs} \\
2-5 \mathrm{yrs} \\
>5 \mathrm{yrs}\end{array}$ & $\begin{array}{l}5(16.7 \%) \\
14(46.7 \%) \\
11(36.7 \%)\end{array}$ & $\begin{array}{l}5(16.7 \%) \\
15(50 \%) \\
10(33.3 \%)\end{array}$ & 0.840 \\
\hline $\begin{array}{ll}\text { Diabetic control } \\
\text { Status }\end{array}$ & $\begin{array}{l}\text { Controlled } \\
\text { Uncontrolled }\end{array}$ & $\begin{array}{ll}7 & (23.3 \%) \\
26 & (76.7 \%)\end{array}$ & $\begin{array}{l}24(80 \%) \\
6(20 \%)\end{array}$ & 0.000 \\
\hline BMI & $\begin{array}{l}\text { Normal } \\
\text { Overweight } \\
\text { Obese }\end{array}$ & $\begin{array}{l}12(40 \%) \\
10(33.3 \%) \\
8(26.7 \%)\end{array}$ & $\begin{array}{l}9(30 \%) \\
15(50 \%) \\
6(20 \%)\end{array}$ & 0.812 \\
\hline
\end{tabular}

There is a significant difference in the control status of Diabetic in compared with the normal subjects. But there is no significant were found in the duration of diabetes and BMI of both control and Diabetic subjects.

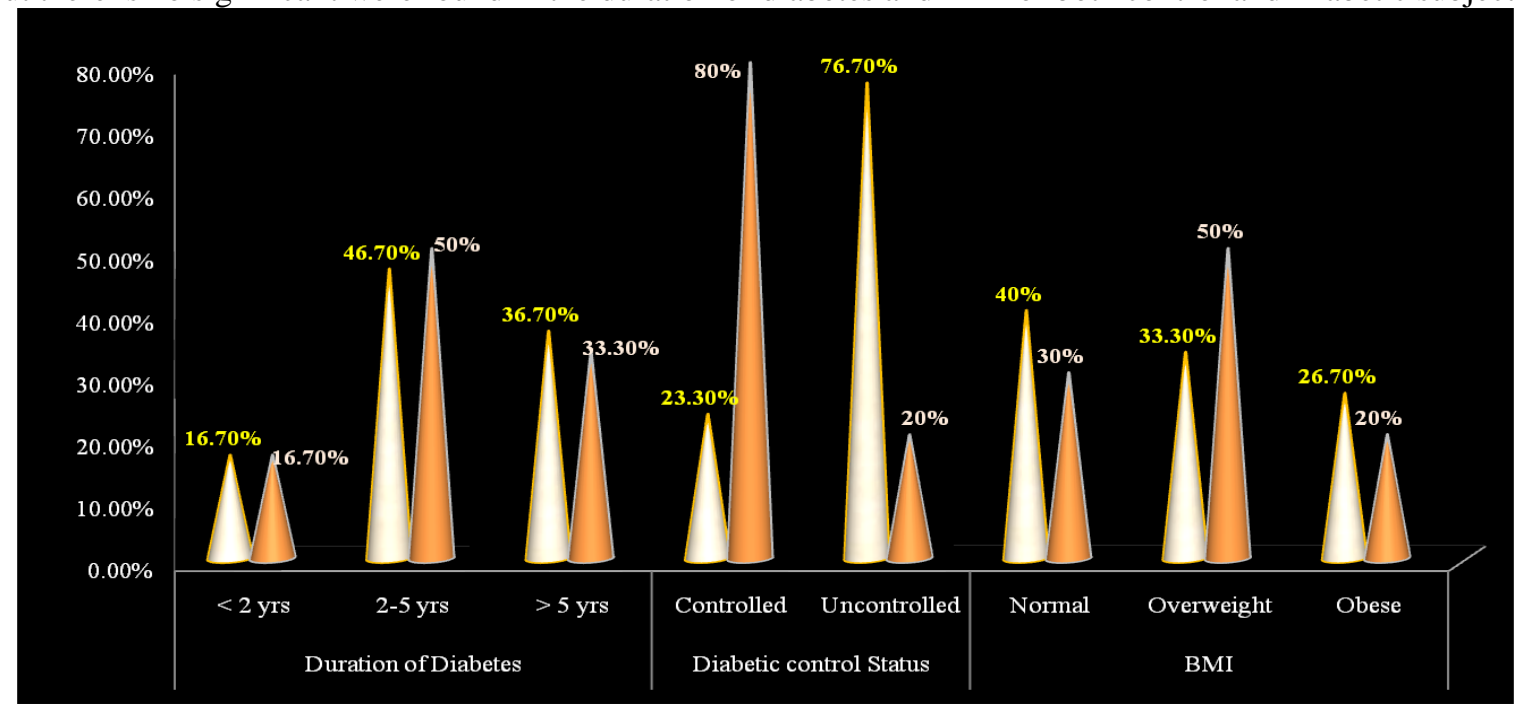




\begin{tabular}{|c|c|c|c|c|}
\hline \multirow{2}{*}{ Risk Factors } & & \multicolumn{2}{|l|}{ Groups } & \multirow{2}{*}{ Significant $P$ value } \\
\hline & & Study Group (30) & Control Group (30) & \\
\hline Systolic BP & $\begin{array}{l}<130 \\
130-150 \\
>150\end{array}$ & $\begin{array}{l}0 \\
11(36.7 \%) \\
19(63.3 \%)\end{array}$ & $\begin{array}{l}2(6.7 \%) \\
10(33.3 \%) \\
18(60 \%)\end{array}$ & 0.651 \\
\hline Diastolic BP & $\begin{array}{l}<90 \\
90-100 \\
>100\end{array}$ & $\begin{array}{l}1(3.3 \%) \\
16(53.3 \%) \\
13(43.3 \%)\end{array}$ & $\begin{array}{l}6(20 \%) \\
14(46.7 \%) \\
10(33.3 \%)\end{array}$ & 0.160 \\
\hline
\end{tabular}

There is no significant were found in the Blood Pressure of Diabetic Patient when compared with normal subjects.

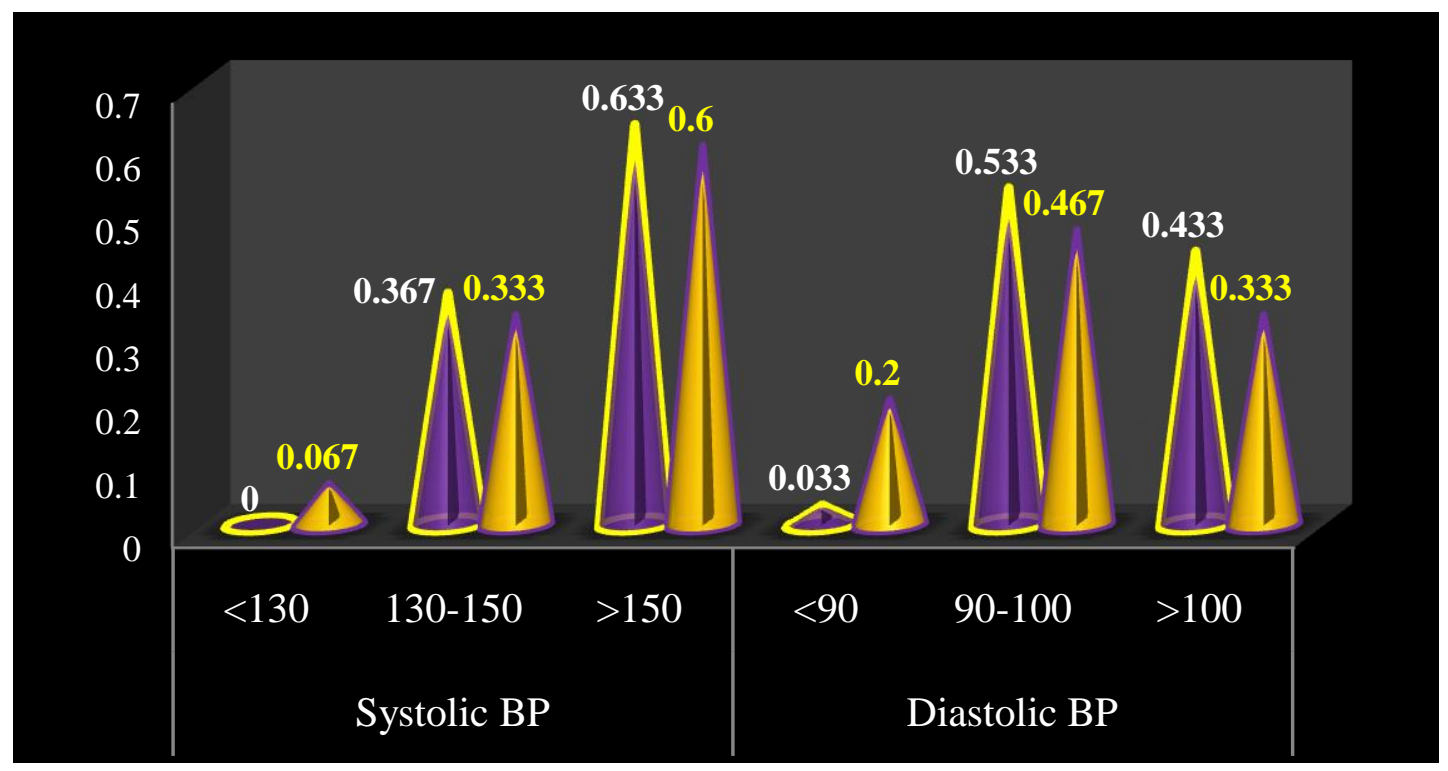

\begin{tabular}{|l|l|l|l|l|}
\hline \multirow{2}{*}{ Risk Factors } & Groups & Chi - Square \\
\cline { 3 - 5 } & Study Group (30) & Control Group (30) & \multirow{2}{*}{0.001} \\
\hline \multirow{2}{*}{ QTC } & Not prolonged & $24(80 \%)$ & $6(20 \%)$ & \\
\cline { 2 - 4 } & Prolonged & $7(23.33 \%)$ & $23(76.67 \%)$ & \\
\hline
\end{tabular}

The QT interval in both patient and controls are presented in the above table. In our findings, the QTc prolongation was more significant in Diabetes when compared to the normal subjects $(\mathrm{P}<0.001)$. 


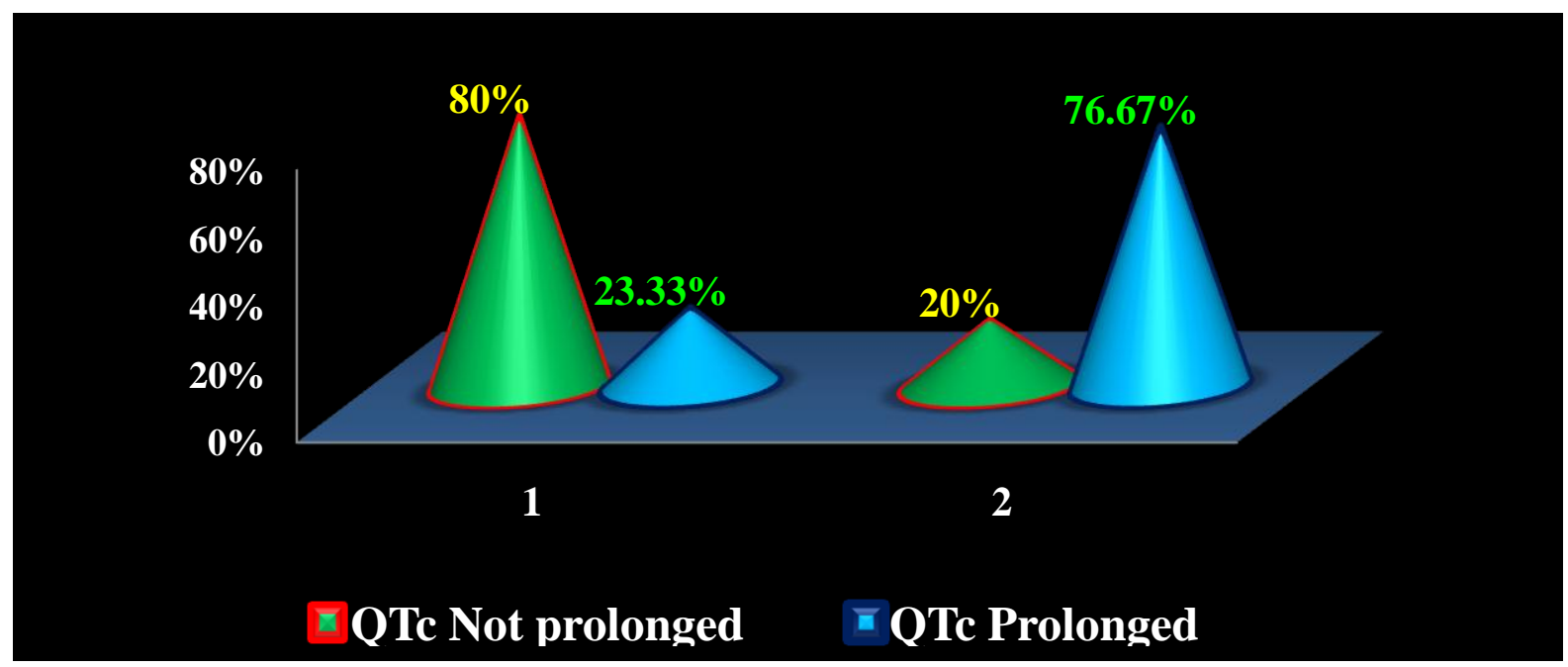

Linear Model:

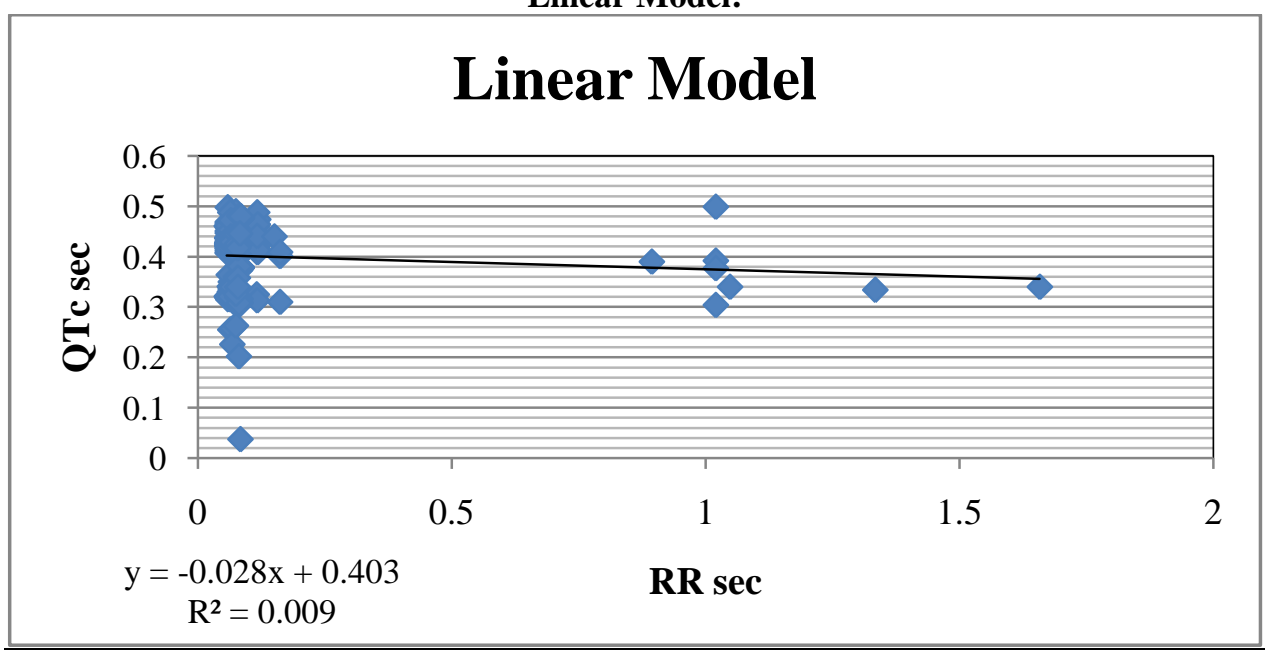

The above QT and RR regression model providing QT interval values that are independent of corresponding RR interval values, which were developed in diabetic subjects. The QT values were mentioned in the above figure shows most of the values are prolonged for the single subjects and QT values were obtained by regression model shows significant with the help of the $\mathrm{R}^{2}$ values $(=0.009)$. When the duration of diabetes was taken into the account, it was more significant for diabetes when compared with normal subjects.

The above table shows that the cardiovascular reflex tests were assessed to the patient with diabetes. Abnormal responses on individual AFT revealed that HRV during deep breathing and Valsalva ratio $(0.99 \pm$ 0.08 and $1.07 \pm 0.09$ respectively) were more significant whereas 30:15 ratio and handgrip test were less significant. SBP response to standing was shown to be significant $(147 \pm 10 \mathrm{mmHg})$ whereas DBP $(86 \pm 10$ $\mathrm{mmHg}$ ) did not show any difference in patient compared with normal subjects.

\section{DISCUSSION}

Qt interval prolongation is an abnormality of the electrical activity of the heart that places individuals at risk for ventricular arrhythmias. The underlying cause of this electrical imbalance may be genetic or induced by extrinsic factors (David et. al. 2005). Although serious complications of QT interval prolongation are rare, the identification of patients at risk may help to prevent these occurrences.

The QT interval represents the duration of the ventricular action potential and is measured from the beginning of depolarization until the end of repolarization. Ventricular depolarization results from the influx of sodium ions across the plasma membrane of cardiac myocytes, whereas ventricular repolarization is controlled by the outward flow of potassium ions. The duration of repolarization is mediated by potassium channels, and the delayed rectifying potassium currents, rapid and slow are particularly important. When the movement of ions across the channels is disturbed and an excess of positively charged ions accumulates inside myocytes, ventricular repolarization is prolonged, resulting in prolongation of the QT interval. 
There is an association of prolonged QT interval with CAN in diabetes mellitus (C. P. Mathur et. al., the exact mechanism of QT prolongation is not. It is generally agreed that parasympathetic have little influence on QT modulations. Some non quantifiable sympathetic imbalance is also responsible for QT prolongation as parasympathetic have little influence on QT modulation. This is due to asymptomatic and debiliating nature of the ANS involvement and increased sudden cardiac death. So its early detection is essential to reduce mortality.

The linear graph represents the standard QT/RR relation. This model was not shown for all the patient data due to the lack of adequate results. The parameters $\alpha$ and $\beta$ values correspond to the maximum zero correction $\left(\mathrm{R}^{2}=0.009\right)$ and it was shown that depending on the individual subjects and they can be different for all patients. A mathematical model to describe the QT/RR relationship can be valid for one patient but not for another due to lack of adequate result and also due to significant intra subject variability. The reason for that may be fact that although changes in Heart Rate and Heart Rate Variability influence changes in QT interval, QT interval variability cannot be entirely explained by changes in the autonomic nervous system. it is likely that beat to beat changes in action potential duration depend on instantaneous changes in the above mentioned channel activity which involved in the repolarisation.

\section{CONCLUSION}

In conclusion, the analysis of QT intervals and its deviations attained by the linear regression model, differed significantly for diabetes with longer duration; if it is further validated with the other regression model, it could be use to predict the increasing risk factor of Cardio autonomic neuropathy.

\section{BIBLIOGRAPHY}

[1]. Abdulkadir K, Zekeriya A, Emin M and Atilla T, "Orthostatic hypotension and heart rate variability as indicators of cardiac autonomic neuropathy in diabetes mellitus", Eur J Gen Med 2005; 2(1); 5-9.

[2]. Chritensen PK, Gall MA, Major-Pedersen A, et al. QTc interval length and QT dispersion as predictors of mortality in patients with non-insulin dependent diabetes mellitus. Scand J Clin Lab Invest 2000;60:323-32.

[3]. Dekker JM, Feskens EJM, Schouten EG, et al. QTc duration is associated with levels of insulin and glucose tolerance. The Zutphen elderly study. Diabetes 1996;45:376-80. Carnethon MR, Anthony MS, Cascio WE, et al. A prospective evaluation of risk of QT prolongation with HRT: the atherosclerosis risk in communities study. Ann Epidemiol 2003;13:530-6.

[4]. Eric a. Whitsel et.,al., Electrocardiographic QT Interval Prolongation and Risk of Primary Cardiac Arrest in Diabetic Patients, Int. J. Diab. Dev. Countries (2005), vol. 25

[5]. Ewing DJ, Boland O, Neilson JM, Cho CG, Clarke BF: Autonomic neuropathy, QT interval lengthening, and unexpected deaths in male diabetic patients.Diabetologia 34:182-185, 1991

[6]. Familoni O. B., Odusan O and Raimi T. H., "The relationship between QT intervals and Cardiac Autonomic Neuropathy in Nigerian Patients with Type 2 Diabetes Mellitus”, Nigerian Medical Prationer, 2008, Vol. 53.

[7]. Hoeldtke RD, Bryner KD, McNeill DR, Hobbs GR, Riggs JE, Warehime SS, Christie I, Ganser G, Van Dyke K: Nitrosative stress, uric acid, and peripheral nerve function in early type 1 diabetes. Diabetes 51:2817-2825, 2002

[8]. Jermendy G, Toth L, Voros P, Koltai MZ, Pogatsa G: Cardiac autonomic neuropathy and QT interval length: a follow-up study in diabetic patients. Acta Cardiol 46:189-200, 1991

[9]. Kristi Veski, Deniss Karai, Kristjan Pilt, Kalju Meigas and Jüri Kaik, "Application of novel QT interval correction and QT/RR assessment models to ECG 24-hour recordings in cardiac patients”, Estonian Journal of Engineering, 2010, 16, 1, 107-120.

[10]. Naas AAO, Davidson NC, Thompson C, et al. QT and QTc dispersion are accurate predictors of cardiac death in newly diagnosed non-insulin dependant diabetes: cohort study BMJ 1998:316:745 - 6.

[11]. Obrosova IG: How does glucose generate oxidative stress in peripheral nerve? Intern Review Neurobiology 50:335,2002

[12]. Oka H, Mochio S, Sato K etal., "Prolongation of QTc interval and nervous dysfunction in diabetic patients", Diabetes Res Clin Pract 1996; 31(1-3); 63-70.

[13]. Sara Giunti etal., "Incidence and Risk Factors of prolonged QTc interval in Type I diabetes", Eurodiab IDDM.

[14]. Sawicki PT, Bender DR, Berger M: Prolonged QT interval as a predictor of mortality in diabetic nephropathy. Diabetologia 39:77-81, 1996

[15]. Sawicki PT, Kiwitt S, Bender R, Berger M: The value of QT interval dispersion for identification of total mortality risk in non-insulin-dependent diabetes mellitus. J Intern Med 243:49-56, 1998

[16]. Takebayashi K, Sugita R, Tayama K, Inakai T. "The connection between QT dispersion and autonomic neuropathy inpatients with type 2 diabetes mellitus", Exp Clin Endocrinol 2003; 111: 351-357.

[17]. Veglio M, Sivieri R, Chinaglia A, Scaglione L, Cavallo-Perin P: QT interval prolongation and mortality in type 1 diabetic patients: a 5-year cohort prospective study: Neuropathy Study Group of the Italian Society of the Study of Diabetes, Piemonte Affiliate. Diabetes Care 23:1381-1383, 2000

[18]. Veglio M, Sivieri R, Chinaglia A, Scaglione L, Cavallo-Perin P: QT interval prolongation and mortality in type 1 diabetic patients: a 5-year cohort prospective study: Neuropathy Study Group of the Italian Society of the Study of Diabetes, Piemonte Affiliate. Diabetes Care 23:1381-1383, 2000

[19]. Veves A, King GL: Can VEGF reverse diabetic neuropathy in human subjects?J Clin Invest 107:1215-1218, 2001

[20]. Vinik AI, Erbas T: Recognizing and treating diabetic autonomic neuropathy.Cleve Clin J Med 68:928-944, 2001 